\title{
Isolation and analysis of $\alpha$-expansin genes in the tree Anthocephalus chinensis (Rubiaceae)
}

\author{
K.X. Ouyang', M.Q. Liu², R.Q. Pian', S.S. Liu² and X.Y. Chen ${ }^{2}$ \\ ${ }^{1}$ Key Laboratory for Genetics and Breeding of Forest Trees and Ornamental Plants, \\ Ministry of Education, National Engineering Laboratory for Forest Tree Breeding, \\ Beijing Forestry University, Beijing, China \\ ${ }^{2}$ State Key Laboratory for Conservation and Utilization of Subtropical Agro-Bioresources, \\ Guangdong Key Laboratory for Innovative Development and \\ Utilization of Forest Plant Germplasm, South China Agriculture University, \\ Guangzhou, China
}

Corresponding author: X.Y. Chen

E-mail: xychen@scau.edu.cn

Genet. Mol. Res. 12 (2): 1061-1073 (2013)

Received February 14, 2012

Accepted August 3, 2012

Published April 10, 2013

DOI http://dx.doi.org/10.4238/2013.April.10.2

\begin{abstract}
Expansins are cell wall-associated proteins that induce wall extension and relax stress by disrupting noncovalent bonds between cellulose microfibrils and cross-linking glycan chains, thereby promoting wall creep. Anthocephalus chinensis is a very fast-growing economically important tree found mainly in South Asia. Sixteen cDNAs, designated AcEXPA1 to AcEXPA16 (GenBank accession Nos. FJ417847, JF922686-JF922700) with corresponding genomic DNA sequences (GenBank accession Nos. GQ228823, JF922701-JF922715), were isolated by amplifying conserved domain binding with genomic walking and RACE techniques from four differential growth tissues in $A$. chinensis. These $\alpha$-expansin homologues were highly conserved in size and sequence; they had the same sequence structures as an $\mathrm{N}$-terminal signal peptide, three exons and two introns. Their amino acid alignment showed that $A$. chinensis expansin genes are divided into three subgroups: A, B and C. This study is the first report on expansin
\end{abstract}


genes from $A$. chinensis. It will be used for a tissue-specific expression model and for studying the relationship between expansin genes, growth rate and wood quality of the xylem in this fast-growing tree.

Key words: $\alpha$-expansin; Phylogenetic tree; Anthocephalus chinensis; Gene cloning

\section{INTRODUCTION}

All plant cells are surrounded by a cell wall that determines their shape, attaches cells together, provides essential mechanical support and rigidity, and also acts as a critical barrier against invading pathogens (O'Malley and Lynn, 2000; Cosgrove, 2000). However, it simultaneously limits cell growth and increase of cell protoplasm. Expansins (EXP) are cell wall proteins that are essential for cell wall extension and stress relaxation (Cosgrove et al., 2002). Although the exact mechanism by which expansins cause loosening and extension of cell walls is still poorly understood, a hypothesis states that expansins break noncovalent bonding, hydrogen bonding between cell wall polysaccharides, thereby allowing turgor-driven polymer creep and stimulating plant growth (McQueen-Mason and Cosgrove, 1994; Hutchison et al., 1999; Cosgrove, 2000; Cosgrove et al., 2002; Lee and Kende, 2002; Gray-Mitsumune et al., 2004, 2008; Wang et al., 2011).

Since the first report of the presence of two expansins in a crude protein extract from the cell walls of cucumber seedling hypocotyls (McQueen-Mason et al., 1992), an increasing number of expansin genes, along with their complete gene sequences, have been isolated from various plants, such as Arabidopsis thaliana, rice (Oryza sativa), poplar (Populus trichocarpa), papaya (Carica papaya), maize (Zea mays), and tobacco (Nicotiana tabacum) (Table 1). These known expansins are divided into four known classes: $\alpha$-expansin (EXPA), $\beta$-expansin (EXPB), expansin-like A (EXPLA), and expansin-like B (EXPLB) (Shcherban et al., 1995; Cosgrove, 2000; Lee et al., 2001; Cosgrove et al., 2002; Kende et al., 2004; Sampedro and Cosgrove, 2005) (http://homes.bio.psu.edu/expansins/genes.htm). Research has mainly focused on the two former expansin classes, whereas expansin-like A and expansin-like B proteins are not well investigated (Lee et al., 2001; Sampedro and Cosgrove, 2005). The data in Table 1 indicate that there are more $\beta$-expansin genes in rice than in dicots, but fewer $\alpha$-expansin genes in maize.

Table 1. Number of expansin genes in six model plants.
\begin{tabular}{lcccccc}
\hline Gene style & \multicolumn{7}{c}{ Plant species } \\
\cline { 2 - 7 } & Arabidopsis & Rice & Poplar & Papaya & Maize & Tobacco \\
\hline EXPA & 26 & 33 & 27 & 15 & 5 & 6 \\
EXPB & 6 & 18 & 3 & 3 & 11 & 0 \\
EXPLA & 3 & 4 & 2 & 1 & 0 & 0 \\
EXPLB & 1 & 1 & 4 & 0 & 0 & 0 \\
\hline
\end{tabular}

$\mathrm{EXPA}=\alpha$-expansin; EXPB $=\beta$-expansin; EXPLA $=$ expansin-like A; EXPLB $=$ expansin-like B. [http://homes.bio. psu.edu/expansins/genes.htm].

A. chinensis, commonly known as kalempayan (Malaysia), laran (Sabah), kaatoan bangkal (Philippines), kalempajan (Indonesia), mau-lettan-she (Burma), and kadam (India), is 
a member of Neolamarckia tribe belonging to the Rubiaceae family and is distributed widely in South Asia (Hsienshui et al., 1999). It has received high praise in the Philippines, where it has been described as "a gem of a tree" (Fox, 1971), and was universally accepted as "a miraculous tree" by the World Forestry Congress in 1972 because of its fast growth. In this context, cloning growth-related genes, AcEXPA, from $A$. chinensis is aimed at understanding the relationship between plant growth and $A c E X P A$ expression.

\section{MATERIAL AND METHODS}

\section{Plant material and tissue sampling}

For RNA extraction, tender leaves and buds were collected from lateral branches, and cambium scrapings were collected from the diameter at breast height of a 4-year-old $A$. chinensis tree according to the method described by Gray-Mitsumune et al. (2004). Young roots were collected from 6-month-old trees cultivated in a greenhouse. All excised samples were immediately frozen in liquid nitrogen, and were then stored at $-80^{\circ} \mathrm{C}$ in a refrigerator until RNA extraction.

\section{DNA extraction, PCR amplification of the central conservative region of $\alpha$-expansin genes and gene walking in genomic DNA}

Total genomic DNA was extracted from the leaves using a DNeasy Plant Kit (Qiagen, China) according to the manufacturer protocol. Comparing the EXPA gene sequences in other plants from NCBI, the degenerate primers [forward: 5'-GG(AGCT)GG(AGCT)GC(AGT) TGTGG(AGT)TA(CT)GG-3', reverse: 5'-TGCCA(AG)TT(CT)TG(AGCT)CCCCA(AG)TT3'] were designed to amplify the central conservative region of EXPA genes, using genomic DNA as the template. PCR was performed in a final reaction volume of $25 \mu \mathrm{L}$ containing 20 ng genomic DNA, 1.25 U DNA polymerase (Takara, Japan), $50 \mathrm{ng}$ forward primer, $50 \mathrm{ng}$ reverse primer, 1X PCR buffer (Takara), and $5 \mathrm{mM}$ each of all four dNTPs (Takara). Touchdown PCR conditions were as follows: $94^{\circ} \mathrm{C}$ hot start for $5 \mathrm{~min} ; 94^{\circ} \mathrm{C}$ denaturation for $30 \mathrm{~s}, 62^{\circ} \mathrm{C}$ annealing for $30 \mathrm{~s}$ with $1^{\circ} \mathrm{C}$ decrease each cycle, and $72^{\circ} \mathrm{C}$ extension for $1.5 \mathrm{~min}$ for 13 cycles; followed by $94^{\circ} \mathrm{C}$ denaturation for $30 \mathrm{~s}, 50^{\circ} \mathrm{C}$ annealing for $30 \mathrm{~s}$, and $72^{\circ} \mathrm{C}$ extension for 1.5 min for 30 cycles, and a final extension at $72^{\circ} \mathrm{C}$ for $10 \mathrm{~min}$.

Genomic walking upstream and downstream PCR primers were subsequently based on the central conservative region sequences as described by the GenomeWalker Kit user manual (www.clontech.com, Takara). Seven restriction endonucleases, AluI, DraI, EcoRI, HaeIII, RsaI, SspI, and StuI, were used for genomic DNA digestion and seven walking libraries were constructed according to the kit.

\section{Total RNA isolation and cDNA synthesis}

Total RNA was extracted using a RNeasy Plant Kit (Qiagen) according to manufacturer instructions. Additional on-column DNase digestions were performed three times during the RNA purification using the RNase-Free DNase Set (Qiagen). RNA was then quantified based on agarose gel electrophoresis and absorption at $260 \mathrm{~nm}$. Quantified RNA was reverse- 
transcribed into cDNA utilizing the PrimeScript II 1st-strand cDNA Synthesis Kit and the supplied polythymine primers (Takara).

\section{Subcloning and sequencing}

All PCR products were resolved by agarose gel electrophoresis, excised, and purified using Ultrafree-DA (Millipore, USA) centrifugal filter units. The purified DNA was ligated into a pMD19-T vector and transformed into DH5 $\alpha$ competent cells. A positive clone for each individual was selected for further sequencing with RV-M and M13-47 primers.

\section{Sequence assembly and open reading frame (ORF) prediction}

The central conservative region sequence, with 5'-upstream and 3'-downstream of EXPA sequences, was assembled with DNAMAN 6.0 (Lynnon BioSoft, Canada). The ORF, intron and exon were predicted by the online tool (http://linux1.softberry.com/berry.phtml?topic=fgenesh \&group=programs\&subgroup=gfind, http://www.cbs.dtu.dk/services/NetGene2). Then, the fulllength EXPA were amplified utilizing a high-fidelity DNA polymerase, PrimeSTAR HS DNA Polymerase (Takara), to confirm the accuracy of the sequences, and the 3'-untranslated region (UTR) sequence was obtained by 3'-Full RACE Core Set Ver.2.0 (Takara).

The actual intron and exon regions were obtained by directly comparing the genomic and transcriptional sequences. The N-terminal signal peptide cleavage sites were predicted using the SignalP program (http://www.cbs.dtu.dk/services/SignalP2.0).

\section{Phylogenetic analysis of $\alpha$-expansins}

Amino acid sequences deduced from the cDNA sequences were aligned by ClustalW with its default setting for protein multiple alignment [weight matrix, Gonnet; gap opening penalty, 10; gap extension penalty, 0.1; residue-specific penalties, ON; hydrophilic penalties, ON; gap separation distance, 4; end gap separation, OFF; delay divergent cutoff (\%), 30] and viewed with BOXSHADE (http://www.ch.embnet.org/software/BOX_form.html). The corresponding alignment of the nucleotide sequences between $A$. chinensis $\alpha$-expansin genes and that of other plants was performed with ClustalW using its default setting for DNA multiple alignment [weight matrix, IUB; gap opening penalty, 15; gap extension penalty, 6.66; use negative matrix, OFF; delay divergent cutoff $(\%), 30]$. A phylogenetic tree was generated from this alignment using the MEGA 4 program with the neighbor-joining method, maximum composite likelihood, bootstrap method, 100 bootstrap replications, and the complete deletion option (Gray-Mitsumune et al., 2004; Tamura et al., 2007). The phylogenetic tree was unrooted.

\section{RESULTS}

\section{Amplification of the conserved domain of $\alpha$-expansin genes}

To establish methods for isolating A. chinensis $\alpha$-expansin genes, we BLASTed most $\alpha$-expansin genes in plants. As an example, the four expansin amino acid sequences, AtEXPA6 (AAB38072), PaEXPA1 (AAC33529), NtEXPA4 (AAC96080), and PtEXPA1 (AY675563), 
demonstrated alignment for conservative regions (Figure 1) using ClustalW. The forward and reverse primers were designed as 5'-GG(AGCT)GG(AGCT)GC(AGT)TGTGG(AGT)TA(CT) GG-3' and 5'-TGCCA(AG)TT(CT)TG(AGCT)CCCCA(AG)TT-3' from "GGACGYG" and "NWGQNWQ", respectively, which are indicated as F and R, respectively, in Figure 1. Then, we used the primers to amplify the conservative region of $A$. chinensis $\alpha$-expansin genes. PCR amplification produced three electrophoretic bands, shown in Figure 2. Thirty positive clones from the PCR products of each vector-ligated band were sequenced, and 16 different sequences were obtained and identified as conservative regions in $\alpha$-expansin genes by BLASTN (http://blast. ncbi.nlm.nih.gov/Blast.cgi?PROGRAM=blastn\&BLAST_PROGRAMS=megaBlast\&PAGE TYPE=BlastSearch\&SHOW_DEFAULTS=on\&LINK_LOC=blasthome).

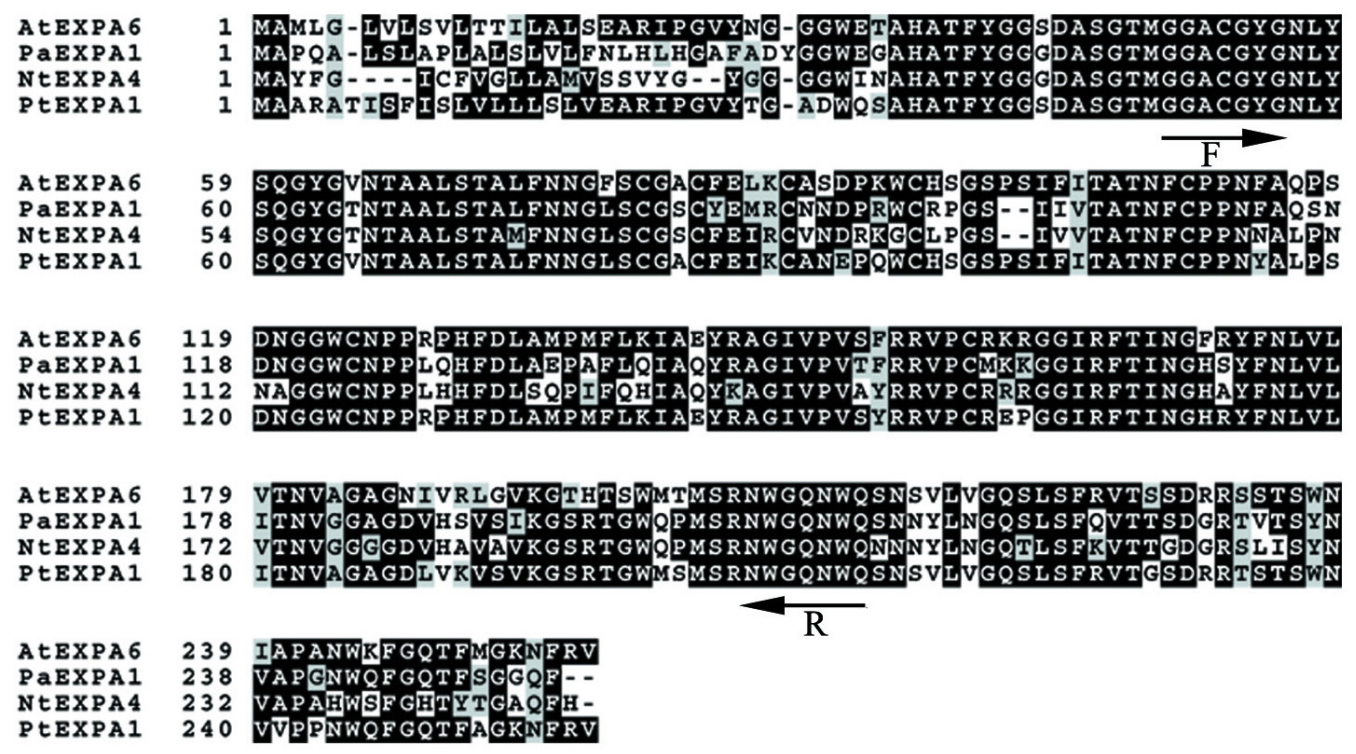

Figure 1. Alignment of AtEXPA6 (AAB38072), PaEXPA1 (AAC33529), NtEXPA4 (AAC96080), and PtEXPA1 (AY675563). The numbers shown at the left of each sequence are the positions of amino acid residues in the corresponding proteins. Identical and similar amino acid residues are shaded with black and gray, respectively. The location and directions of primers are indicated by arrows.

\section{Isolation of $A c E X P A 1-16$ full-length cDNA sequences}

In order to amplify the full-length of the genes, genomic walking and RACE (rapid amplification of cDNA ends) were adopted based on the fragment sequences. We eventually isolated 16 A. chinensis $\alpha$-expansin genes designating them AcEXPA1-16 according to the nomenclature of Kende et al. (2004). Detailed information of AcEXPA1-16, including full-length base number, ORF base number, length of predicted amino acid, and signal peptide length, is provided in Table 2. The total amino acid length of AcEXPA1-16 ranged from 240 to 258, whereas their signal peptide length ranged from 19 to 28 amino acids. All AcEXPA1-16 introns had the nucleotides GT at the 5'-splice site and AG at the 3'-splice site, except intron 2 in AcEXPA4, which had TA at the 5'-splice site and GA at the 3'-splice site and neither belonged to the U2-type 
intron (GT-AG) (Simpson and Filipowicz, 1996) nor the U12-type intron (AT-AC) (Levine and Durbin, 2001; Patel and Steitz, 2003). Additionally, $\alpha$-expansin sequences from several different tissues were isolated and showed that most sequences obtained were completely identical except for two, two, and one silent mutations in the AcEXPA1, AcEXPA5, and AcEXPA10 ORF, respectively (Figure 3A-C), one sense mutation in the AcEXPA5 ORF with a C-to-G substitution, which results in the amino acid proline changing to alanine (Figure 3B). Although several reports have shown that mutations might lead to phenotypic alteration as well as change in the molecular function of the genes (Strauss, 2000; Petry and Loos, 2005; Yang and Nielsen, 2008), in the case of our findings, we do not yet know what the consequences of these mutations are.

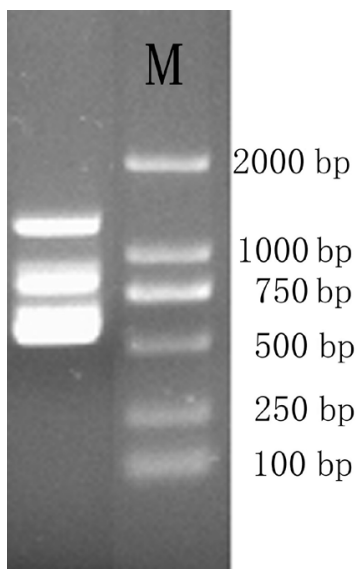

Figure 2. PCR product with the degenerated primers. Left column = PCR products with three bands; right column = molecular marker with number labeled at the right.

Table 2. Detailed information of AcEXPA1-16, including ORF length, length of the predicted protein, signal peptide length, and intron splice site base model.

\begin{tabular}{|c|c|c|c|c|c|c|c|}
\hline \multirow[t]{3}{*}{ Gene name } & \multirow{3}{*}{$\begin{array}{l}\text { ORF length } \\
\quad(b p)\end{array}$} & \multirow{3}{*}{$\begin{array}{l}\text { Amino acid } \\
\text { length }(\mathrm{AA})\end{array}$} & \multirow{3}{*}{$\begin{array}{c}\text { Signal peptide (AA) } \\
\text { (http://www.cbs.dtu.dk/services/SignalP/) }\end{array}$} & \multicolumn{4}{|c|}{ Intron in ORF } \\
\hline & & & & \multicolumn{2}{|c|}{ Intron 1} & \multicolumn{2}{|c|}{ Intron 2} \\
\hline & & & & 5'-terminal & 3'-terminal & 5'-terminal & 3'-terminal \\
\hline AcEXPA1 & 777 & 258 & 21 & GT & AG & GT & AG \\
\hline AcEXPA2 & 768 & 255 & 28 & GT & AG & GT & AG \\
\hline AcEXPA3 & 768 & 255 & 28 & GT & $\mathrm{AG}$ & GT & AG \\
\hline AcEXPA4 & 759 & 252 & 25 & GT & AG & $\mathrm{TA}$ & GA \\
\hline AcEXPA5 & 777 & 258 & 21 & GT & AG & GT & AG \\
\hline AcEXPA6 & 762 & 253 & 26 & GT & AG & GT & AG \\
\hline AcEXPA7 & 747 & 248 & 20 & GT & $\mathrm{AG}$ & GT & AG \\
\hline AcEXPA & 771 & 256 & 19 & GT & AG & GT & AG \\
\hline AcEXРА & 777 & 258 & 22 & GT & AG & GT & AG \\
\hline AcEXPA10 & 723 & 240 & 20 & GT & AG & GT & AG \\
\hline AcEXPA11 & 753 & 250 & 22 & GT & $\mathrm{AG}$ & GT & AG \\
\hline AcEXPA12 & 747 & 248 & 20 & GT & AG & GT & AG \\
\hline AcEXPA13 & 747 & 248 & 20 & GT & AG & GT & AG \\
\hline AcEXPA14 & 741 & 246 & 20 & GT & AG & GT & AG \\
\hline AcEXPA15 & 744 & 247 & 20 & GT & $\mathrm{AG}$ & GT & AG \\
\hline AcEXPA16 & 768 & 255 & 23 & GT & $\mathrm{AG}$ & GT & $\mathrm{AG}$ \\
\hline Eukaryote & & & & GT & AG & GT & AG \\
\hline
\end{tabular}




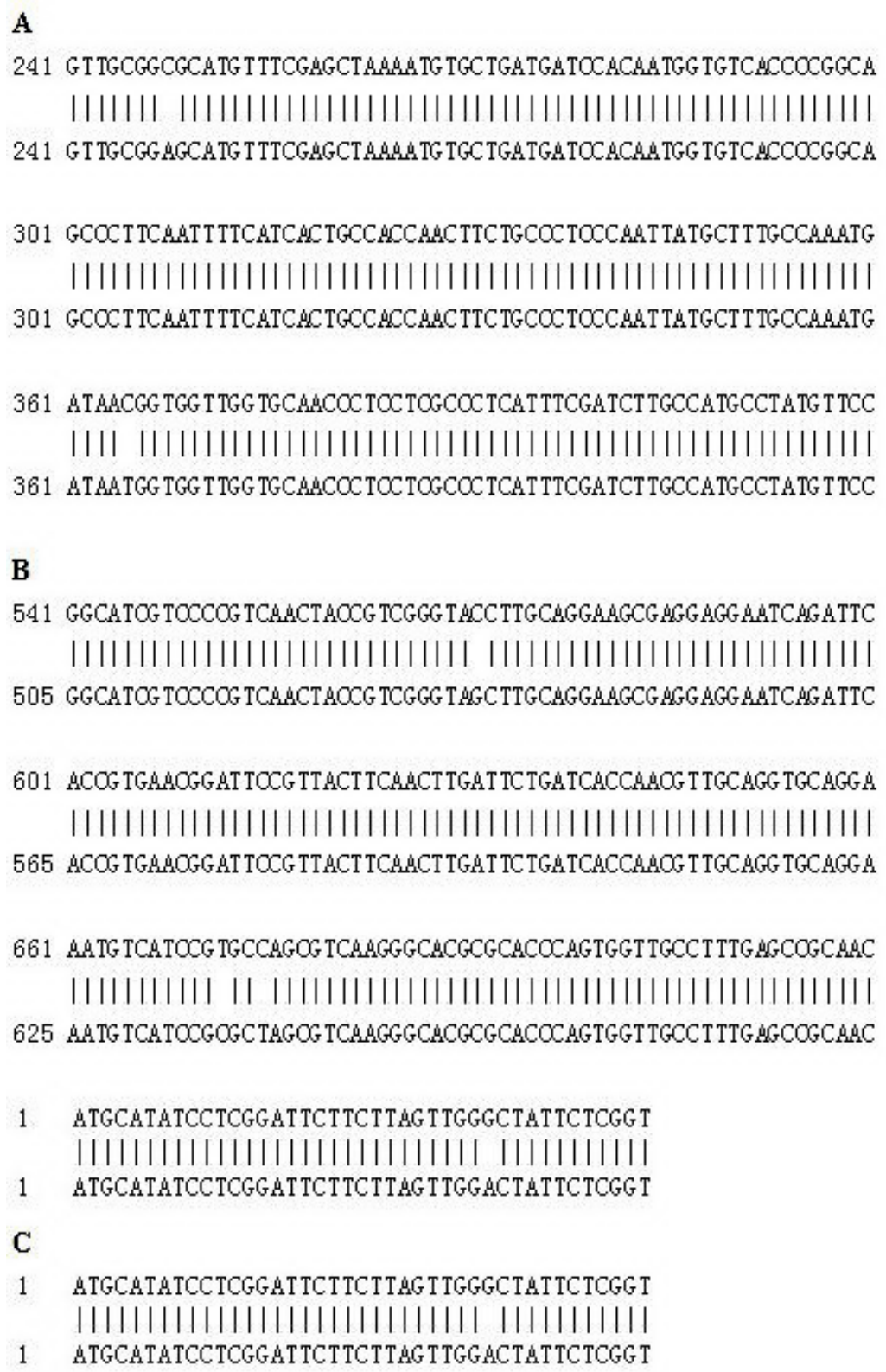

Figure 3. Mutation in AcEXP1, AcEXP5 and AcEXPA10 ORF. A. AcEXP1, B. AcEXP5, C. AcEXPA10.

\section{Intron-exon model of $A c E X P A 1-16$}

To learn how the intron/exon structure changed during the evolution of the expansin gene family, the genomic structure of the cDNA of each AcEXPA family member was com- 
pared and the intron/exon organization of each gene was determined (Figure 4). A schematic representation of the genomic organization of the AcEXPA family members, depicting the number and location of introns within the AcEXPA transcriptional region, is shown in Figure 2. We also found that the intron-exon structure of AcEXPA1-16 is similar to the model of Arabidopsis $\alpha$-expansin genes, where most of the genes are disrupted by introns 1 and 2 (https:// homes.bio.psu.edu/expansins/arabidopsis.htm, Figure 2) (Sampedro and Cosgrove, 2005). Although we detected extreme conservation in the coding sequences and positions of intron/ exon boundaries of all AcEXPA genes, the sizes and sequences of the introns in the coding region and 3'-UTR were found to be significantly divergent among the 16 AcEXPA genes.

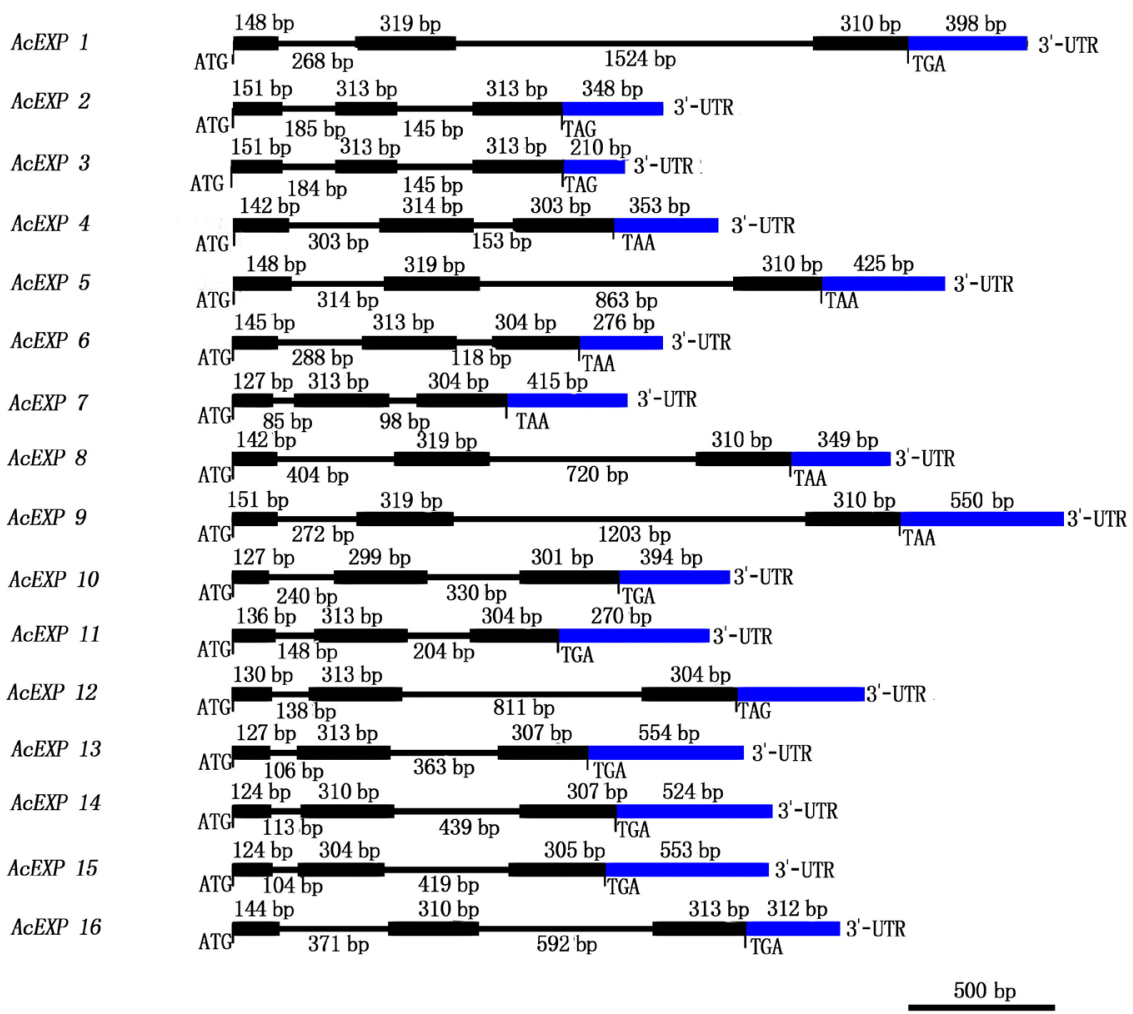

Figure 4. Schematic representation of the structure of the EXPA family in Anthocephalus chinensis with the corresponding gene name. Exons and UTRs are indicated as black and blue boxes, respectively, and lines between boxes are introns. The start code, stop code and length of fragment are labeled in the figure.

\section{Amino acid sequence alignment}

To investigate the similarity of $A$. chinensis $\alpha$-expansin genes, the amino acids of the genes were compared. The coding sequences were deduced from cDNA sequences, and then aligned by ClustalW. Figure 5 illustrates the alignment of the $16 \alpha$-expansin genes. The genes contain key features of expansin, including $\mathrm{N}$-terminal signal peptides, a series of conserved 
C (cysteine, Cys) residues similar to glycoside hydrolase family 45 (GH45) proteins, an HFD (histidine-phynylalanine-aspartate, His-Phe-Asp) motif, which is speculated to form part of the active site, and four $\mathrm{W}$ (tryptophan, Trp) residues near the $\mathrm{C}$ terminus that might be involved in the interaction with cellulose (Shcherban et al., 1995; Sampedro and Cosgrove, 2005), although there is leucine (L) instead of $\mathrm{W}$ at the first $\mathrm{W}$ site in AcEXPA12.

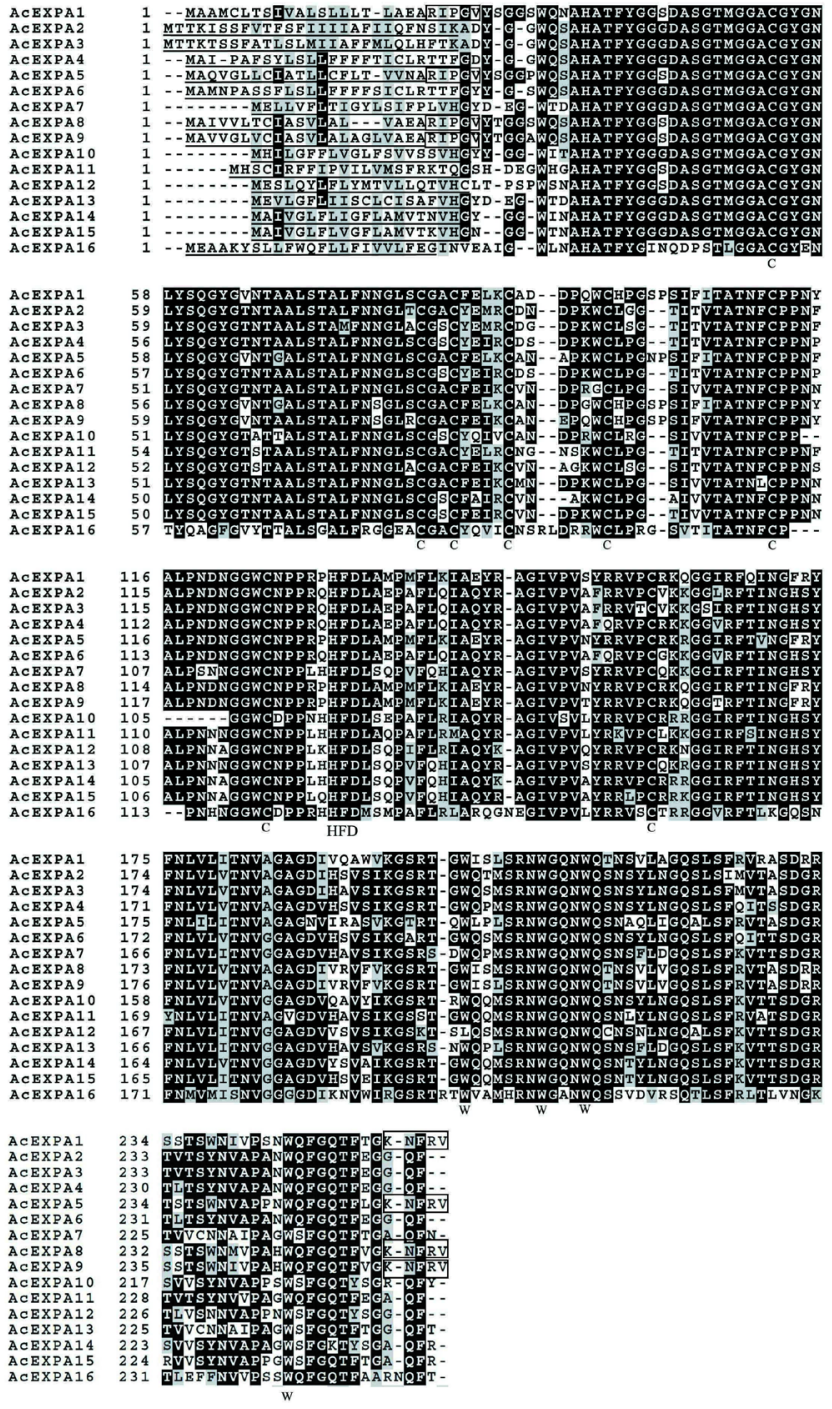

Figure 5. Alignment of the predicted protein sequences of AcEXPA1-16. The N-terminal signal peptides stablished by the SignalP program (www.cbs.dtu.dk/services/SignalP/) are underlined. The conserved Cys, Trp residues and His-Phe-Asp motif residues are indicated by the letters C, W, and HFD, respectively. RIPGV and KNFRV motifs at both the $\mathrm{N}$ and $\mathrm{C}$ ends of mature proteins are boxed. Identical and similar amino acids are represented by black and gray boxes, respectively. Gaps are shown as dashes. Multiple alignments were done by CLUSTAL-W and viewed with the BOXSHADE program. 


\section{Phylogenetic analysis of $\alpha$-expansin genes}

The $\alpha$-expansin gene family splits into four major phylogenetic branches (Figure 6) (Cosgrove, 1998; Link and Cosgrove, 1998; Gray-Mitsumune et al., 2004). Four expansin genes, AcEXPA1, AcEXPA5, AcEXPA8, and AcEXPA9, aligned with subgroup A expansin genes previously described by Link and Cosgrove (1998) and Gray-Mitsumune et al. (2004). The predicted proteins from this subgroup exhibit unique sequence conservation, namely near the $\mathrm{C}$ terminus the amino acid sequence immediately after the predicted signal peptide cleavage site RIPGV, and the C-terminal sequence KNFRV (Figure 5). These two motifs can serve as signature sequences of subgroup A, which is abundant in secondary xylem (Gray-Mitsumune et al., 2004; Wang et al., 2011). Nevertheless, the tissue-specific expressions of these four corresponding genes in A. chinensis are unclear. AcEXPA2, AcEXPA3, AcEXPA4, AcEXPA6, and AcEXPA11 belong to subgroup B, and AcEXPA7, AcEXPA10, AcEXPA12, AcEXPA13, AcEXPA14, and AcEXPA15 belong to subgroup C. Phylogenetic analysis showed that AcEXPA16 belongs neither to any of the above-mentioned subgroups nor to subgroup D in the phylogenetic tree.

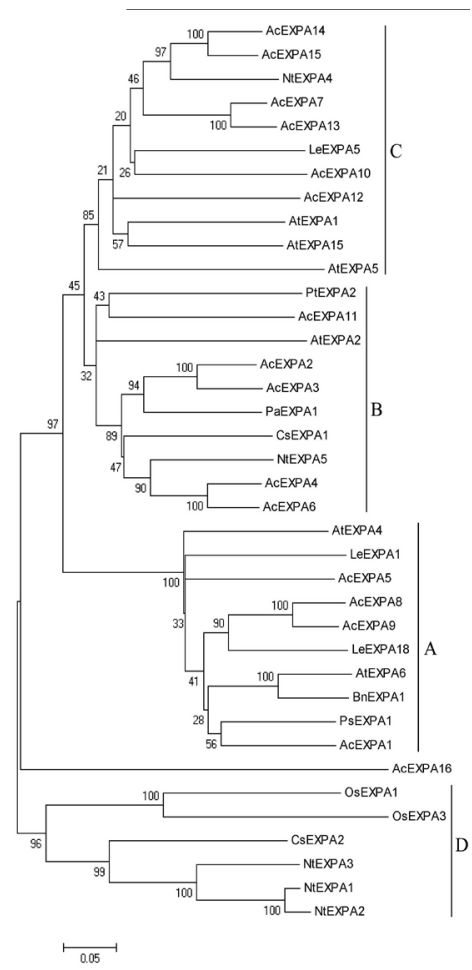

Figure 6. Phylogenetic analysis of $\alpha$-expansin genes. Subgroups A to D as defined by Link and Cosgrove (1998). GenBank accession numbers of expansin genes from Arabidopsis thaliana: AtEXPA1 (U30476), AtEXPA2 (U30481), AtEXPA4 (129526), AtEXPA5 (U30478), AtEXPA6 (U30480), AtEXPA15 (126361); Brassica napus: BnEXPA1 (AJ000885); Cucumis sativus: CsEXPA1 (U30382), CsEXPA2 (U30460); Lycopersicon esculentum: LeEXPA1 (U82123), LeEXPA5 (AF059489), LeEXPA18 (AJ004997); Nicotiana tabacum: NtEXPA1 (AF049350), NtEXPA2 (AF049351), NtEXPA3 (AF049352), NtEXPA4 (AF049353), NtEXPA5 (AF049354); Oryza sativa: OsEXPA1 (Y07782), OsEXPA3 (U30479); Prunus armeniaca: PaEXPA1 (U93167); Pisum sativum: PsEXPA1 (X85187); Pinus taeda: PtEXPA2 (U64890). The tree was unrooted. 


\section{DISCUSSION}

To date, model plants such as Arabidopsis, rice, and poplar have been fully sequenced. Therefore, it is very easy to clone target genes in these species. However, due to limited genomic information, it is still very difficult to clone the genes of interest in most plants, such as A. chinensis, to carry out further research. In this report, we present an efficient method carried out by cloning the conserved motif first, then amplifying full-length genes by RACE and genomic walking to clone genes in this unknown species.

The genomes of model plants contain many $\alpha$-expansin genes (https://homes.bio.psu. edu/expansins/genes.htm). Despite the deduction that the mechanisms of all these $\alpha$-expansins are similar, whether their functions are different is still not entirely clear (McQueen-Mason and Cosgrove, 1994; Cosgrove, 2000; Cosgrove et al., 2002), as various genes are expressed at different levels in different organs or tissues, and also at different development stages (Cho and Cosgrove, 2002; Hiwasa et al., 2003; Gray-Mitsumune et al., 2004; Ishimarua et al., 2007; Sharova, 2007; Figueroa et al., 2009; Jieun et al., 2010). In the present study, we cloned 16 $\alpha$-expansin genes in A. chinensis. This suggests that they might have different functions in cell wall extension. It is also possible that their expression and function are redundant and synergistic, as all $A$. chinensis $\alpha$-expansins exhibited a high level of conservation not only in protein length, but also as specific amino acids, including eight $\mathrm{C}$ residues at the $\mathrm{N}$ terminus, the HFD motif, and four $\mathrm{W}$ residues at the $\mathrm{C}$ terminus (Figure 5) (Shcherban et al., 1995). A. chinensis $\alpha$-expansins might also be regarded as a gene resource for plant molecular engineering.

$\alpha$-expansins possess highly conserved motifs, not only in Arabidopsis and rice, but also in A. chinensis and other species. Thus, the degenerate primers described in this manuscript can also be used to amplify $\alpha$-expansins in other species. Additionally, conservative $\alpha$-expansins might serve as molecular markers to study plant genomic duplication and evolution.

Due to the 3'-oligo(dT) primer limit, an erroneous PCR product might sometimes be produced in the 3'-UTR region. For example, the length of the 3'-UTR containing PolyA of AcEXPA1 and AcEXPA5 in our first PCR products was much shorter than that of the other AcEXPA genes, being 121- and 42-bp long, respectively. These 3'-UTR lengths were much shorter than those of Arabidopsis (http://www.ncbi.nlm.nih.gov/nucleotide). This illustrates the fact that they were not complete sequences containing PolyA. Therefore, downstream primers were designed with genomic DNA sequences following the false PolyA for amplification of their cDNA, cooperating with upstream primers. The sequences of the PCR products were aligned with prior 3'-UTR sequences. The result is shown in Figure 7. The false PolyA is basically consistent with the sequence of the corresponding site in the second PCR product (Figure 7). This indicates that the oligo(dT) with adaptor primer was annealed at the site possessing a tandem A base in front of the PolyA in the mRNA in RT-PCR of 3'-RACE, thereby producing the short 3'-UTR.

Phylogenetic analysis showed that $\alpha$-expansin genes fall into four clades (A-D; Figure 5) similar to those previously described by Link and Cosgrove (1998). It has previously been postulated that the $\alpha$-expansins within each clade may act in a manner similar to each other (Link and Cosgrove, 1998). In the phylogenetic tree, AcEXPA1, AcEXPA5, AcEXPA8, and AcEXPA9 aligned within subgroup $A$, which contains several expansin genes expressed in primary and secondary vascular tissues, such as the AtEXPA4, AtEXPA6 (S. Turner, data published online at http://affymetrix.arabidopsis.info/narrays/experimentbrowse.pl), and LeEXPA18 genes (Reinhardt et al., 1998). All of the subgroup proteins have common conserved domain as RIPGV after the predicted signal peptide cleavage site and KNFRV at the C terminal. AcEXPA2, 


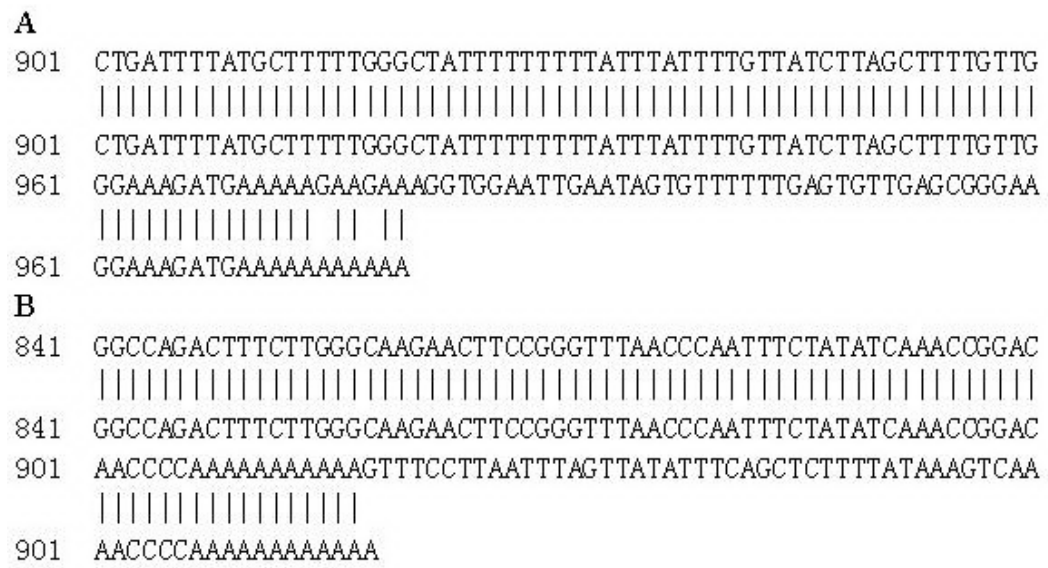

Figure 7. Sequences of PCR production with the downstream primers after the false PolyA were aligned with prior cDNAs. Upper line is sequence of second-time PCR production and lower line is cDNA sequence with false PolyA. A. AcEXPA1; B. AcEXPA5.

AcEXPA3, AcEXPA4, AcEXPA6, and AcEXPA11 belong to subgroup B, which contains genes expressed in growing roots and hypocotyls. Members of this subgroup, such as PtEXPA2, are expressed during adventitious root formation and in the base of the hypocotyl in response to indole-3-butyric acid treatment in loblolly pine (Pinus taeda) (Hutchison et al., 1999), and CsEXPA1, expressed in cucumber hypocotyl (Shcherban et al., 1995). Other $A$. chinensis genes, AcEXPA7, AcEXPA10, AcEXPA12, AcEXPA13, AcEXPA14, and AcEXPA15, belong to subgroup $\mathrm{C}$, which includes Arabidopsis AtEXPA1 and AtEXPA5, and tomato LeEXPA5. AtEXPA1 is known to express and accelerate stomatal opening in Arabidopsis (Zhang et al., 2011), while AtEXPA5 is mainly expressed in the aerial parts of Arabidopsis, especially in the inflorescence stems and flowers (Park et al., 2010). Another member of subgroup C, LeEXPA5, has been shown to be expressed in expanding fruit, with expression levels being highest in full-size maturing green fruit and which decline during the early stages of ripening in tomato (Brummell et al., 1999).

\section{ACKNOWLEDGMENTS}

We thank Professor Ai-min Wu for revising the manuscript. Research supported by the special funds project of Forestry Public Welfare Industry Research of China (\#201004020).

\section{REFERENCES}

Brummell DA, Harpster MH and Dunsmuir P (1999). Differential expression of expansin gene family members during growth and ripening of tomato fruit. Plant Mol. Biol. 39: 161-169.

Cho HT and Cosgrove DJ (2002). Regulation of root hair initiation and expansin gene expression in Arabidopsis. Plant Cell 14: 3237-3253.

Cosgrove DJ (1998). Cell Wall Loosening by Expansins. Plant Physiol. 118: 333-339.

Cosgrove DJ (2000). Loosening of plant cell walls by expansins. Nature 407: 321-326.

Cosgrove DJ, Li LC, Cho HT, Hoffmann-Benning S, et al. (2002). The growing world of expansins. Plant Cell Physiol. 43: 1436-1444.

Figueroa CR, Pimentel P, Dotto MC, Civello PM, et al. (2009). Expression of five expansin genes during softening of 
Fragaria chiloensis fruit: effect of auxin treatment. Postharvest Biol. Tec. 53: 51-57.

Fox JED (1971). Anthocephalus chinensis, the Laran Tree of Sabah. Econ. Bot. 25: 221-233.

Gray-Mitsumune M, Mellerowicz EJ, Abe H, Schrader J, et al. (2004). Expansins abundant in secondary xylem belong to subgroup A of the alpha-expansin gene family. Plant Physiol. 135: 1552-1564.

Gray-Mitsumune M, Blomquist K, McQueen-Mason S, Teeri TT, et al. (2008). Ectopic expression of a wood-abundant expansin PttEXPA1 promotes cell expansion in primary and secondary tissues in aspen. Plant Biotechnol. J. 6: 62-72.

Hiwasa K, Rose JK, Nakano R, Inaba A, et al. (2003). Differential expression of seven alpha-expansin genes during growth and ripening of pear fruit. Physiol. Plant 117: 564-572.

Hsienshui L, Wancheung K, Chen WC and Hsianghao H (1999). Flora. In: Rubiaceae (Hsienshui L, ed.). Science Press, Beijing, 260-261.

Hutchison KW, Singer PB, McInnis S, Diaz-Sala C, et al. (1999). Expansins are conserved in conifers and expressed in hypocotyls in response to exogenous auxin. Plant Physiol. 120: 827-832.

Ishimaru M, Smith DL, Gross KC and Kobayashi S (2007). Expression of three expansin genes during development and maturation of Kyoho grape berries. J. Plant Physiol. 164: 1675-1682.

Jieun J, O'Donoghue EM, Dijkwel PP and Brummell DA (2010). Expression of multiple expansin genes is associated with cell expansion in potato organs. Plant Sci. 179: 77-85.

Kende H, Bradford K, Brummell D, Cho HT, et al. (2004). Nomenclature for members of the expansin superfamily of genes and proteins. Plant Mol. Biol. 55: 311-314.

Lee Y and Kende H (2002). Expression of alpha-expansin and expansin-like genes in deepwater rice. Plant Physiol. 130: 1396-1405.

Lee Y, Choi D and Kende H (2001). Expansins: ever-expanding numbers and functions. Curr. Opin. Plant Biol. 4: 527-532.

Levine A and Durbin R (2001). A computational scan for U12-dependent introns in the human genome sequence. Nucleic Acids Res. 29: 4006-4013.

Link BM and Cosgrove DJ (1998). Acid-growth response and alpha-expansins in suspension cultures of bright yellow 2 tobacco. Plant Physiol. 118: 907-916.

McQueen-Mason S and Cosgrove DJ (1994). Disruption of hydrogen bonding between plant cell wall polymers by proteins that induce wall extension. Proc. Natl. Acad. Sci. U. S. A. 91: 6574-6578.

McQueen-Mason S, Durachko DM and Cosgrove DJ (1992). Two endogenous proteins that induce cell wall extension in plants. Plant Cell 4: 1425-1433.

O’Malley RC and Lynn DG (2000). Expansin message regulation in parasitic angiosperms: marking time in development. Plant Cell 12: 1455-1465.

Park CH, Kim TW, Son SH, Hwang JY, et al. (2010). Brassinosteroids control AtEXPA5 gene expression in Arabidopsis thaliana. Phytochemistry 71: 380-387.

Patel AA and Steitz JA (2003). Splicing double: insights from the second spliceosome. Nat. Rev. Mol. Cell Biol. 4: 960-970.

Petry F and Loos M (2005). Common silent mutations in all types of hereditary complement C1q deficiencies. Immunogenetics 57: 566-571.

Reinhardt D, Wittwer F, Mandel T and Kuhlemeier C (1998). Localized upregulation of a new expansin gene predicts the site of leaf formation in the tomato meristem. Plant Cell 10: 1427-1437.

Sampedro J and Cosgrove DJ (2005). The expansin superfamily. Genome Biol. 6: 242.

Sharova E (2007). Expansins: Proteins involved in cell wall softening during plant growth and morphogenesis. Russ. $J$. Plant Physl. 54: 713-727.

Shcherban TY, Shi J, Durachko DM, Guiltinan MJ, et al. (1995). Molecular cloning and sequence analysis of expansions - a highly conserved, multigene family of proteins that mediate cell wall extension in plants. Proc. Natl. Acad. Sci. U. S. A. 92: 9245-9249.

Simpson GG and Filipowicz W (1996). Splicing of precursors to mRNA in higher plants: mechanism, regulation and subnuclear organisation of the spliceosomal machinery. Plant Mol. Biol. 32: 1-41.

Strauss BS (2000). Role in tumorigenesis of silent mutations in the TP53 gene. Mutat. Res. 457: 93-104.

Tamura K, Dudley J, Nei M and Kumar S (2007). MEGA4: Molecular Evolutionary Genetics Analysis (MEGA) software version 4.0. Mol. Biol. Evol. 24: 1596-1599.

Wang G, Gao Y, Wang J, Yang L, et al. (2011). Overexpression of two cambium-abundant Chinese fir (Cunninghamia lanceolata) alpha-expansin genes ClEXPA1 and ClEXPA2 affect growth and development in transgenic tobacco and increase the amount of cellulose in stem cell walls. Plant Biotechnol. J. 9: 486-502.

Yang Z and Nielsen R (2008). Mutation-selection models of codon substitution and their use to estimate selective strengths on codon usage. Mol. Biol. Evol. 25: 568-579.

Zhang XQ, Wei PC, Xiong YM, Yang Y, et al. (2011). Overexpression of the Arabidopsis alpha-expansin gene AtEXPA1 accelerates stomatal opening by decreasing the volumetric elastic modulus. Plant Cell Rep. 30: 27-36. 